\title{
The right ventricle: always normal in normal subjects?
}

\author{
E. E. van der Wall
}

Published online: 10 December 2014

(C) The Author(s) 2014. This article is published with open access at Springerlink.com

Right ventricular (RV) structure and function serve as important indicators of a wide spectrum of heart diseases such as congenital heart disease, pulmonary hypertension, hypertrophic cardiomyopathy and arrhythmogenic right ventricular cardiomyopathy/disease (ARVC/D) [1-3]. Over the past years cardiac magnetic resonance imaging (CMR) has proven itself as the preferred imaging modality of choice to evaluate RV anatomy, structure, texture, motion and function [4-9]. In the study by Quick et al. [10], published in the current issue of the Netherlands Heart Journal, a quantitative analysis of normal variations in RV wall motion was evaluated in healthy subjects using CMR. The study population consisted of 65 consecutive patients referred for the evaluation of cardiac function by 3 Tesla-CMR. All subjects were shown to be free of heart disease based on the currently available guidelines for CMR. Balanced steady-state free-precession images were obtained and regional RV wall motion was evaluated and classified based on a standardised segmental model for the right ventricle. In 59 out of 65 subjects ( $>90 \%$ ) wall motion abnormalities of the right ventricle were observed. Wall motion abnormalities were predominantly seen in the apico-lateral segments $(72 \%)$ when compared with the medio-lateral $(24 \%)$ and infero-lateral segments (4\%). Dyskinesia was the most frequent wall motion disorder (62\%), followed by hypokinesia (21\%) and bulging (17\%). The authors concluded that RV wall motion abnormalities are common in subjects supposed to be normal, indicating that one should be aware of the notion that non-pathological wall motion disorders can easily be mistaken for a pathological regional wall motion abnormality, particularly in patients with ARVC where -

E. E. van der Wall $(\bowtie)$

Holland Heart House/Netherlands Society of Cardiology,

Moreelsepark 1, 3511 EP Utrecht, the Netherlands

e-mail: eevanderwall@hotmail.com according to the authors- to date, clear wall motion criteria are lacking.

The study is certainly very interesting in the sense that the right ventricle in normal subjects does not always appear normal when visualised by CMR. Especially, regional wall motion abnormalities are predominantly seen in the apicolateral segments. However, the study warrants the following three comments. First, also acknowledged by the authors, the study was based on a small patient population. As a result, the description of normal variations in RV morphology as observed by CMR are not necessarily absolute and should only be regarded as hypothesis-generating for studying larger patient samples. Second, according to the authors, there is supposedly no in-depth knowledge on RV motion in healthy subjects. This is an obvious understatement as already in 2004 Pennell's group (London, UK) showed that CMR demonstrated good inter-study reproducibility for RV function parameters in 20 healthy subjects, in 20 patients with heart failure, and in 20 patients with hypertrophy, suggesting that CMR is reliable for serial RV assessment [11]. In 2008, Youssef et al. [12] showed in 21 healthy subjects that the strain-encoding CMR technique allowed for rapid quantification of RV regional function with low intra- and inter-observer variability, permitting accurate quantification of regional strain in patients with RV dysfunction. Rather recently, Von Knobelsdorff-Brenkenhoff et al. [13], in nine healthy subjects, showed that fast-gradient echo cine imaging of the RV at 7 Tesla-CMR was feasible and provided good image quality; RV dimensions and function were comparable with steady-state free-precession at 1.5 Tesla-CMR as gold standard. Doesch et al. [14] showed in 20 healthy subjects that CMR-derived measurement of the tricuspid annular plane systolic excursion with a reference point outside the ventricle (TAPSEout) might be used for screening RV motion; however, the detection of subtle changes in $\mathrm{RV}$ function requires the $3 \mathrm{D}$ volumetric CMR approach. Third, and most importantly, Quick et al. 
[10] rather firmly state, that -particularly in ARVC patients- 'to date clear wall motion criteria are lacking'. However, the revised ARVC/D diagnostic Task Force Criteria, already dating from 2010, incorporate clear CMR-defined cut-off values for $\mathrm{RV}$ ejection fraction and RV end-diastolic volume for ARVC [3, 15]. More specifically, the 2010 ARVC/D Task Force Criteria include the following parameters for CMR: 1) regional RV akinesia or dyskinesia or dyssynchronous RV contraction, 2) ratio of RV end-diastolic volume to $\mathrm{BSA} \geq 110 \mathrm{~mL} / \mathrm{m} 2$ (male) or $\geq 100 \mathrm{~mL} / \mathrm{m} 2$ (female), or RV ejection fraction $\leq 40 \%$. Used in this way, CMR-derived RV ejection fraction can help in distinguishing ARVC/D from both normal subjects and/or from the physiological cardiac adaptation in athletes [3, 15].

To summarise, despite the above-mentioned critical comments, the study by Quick et al. [10] is a valuable contribution to our understanding of CMR-derived RV function in normal subjects. The study clearly shows that RV wall motion abnormalities may occur both in normal subjects and in diseased patients, in particular those with ARVC. Fortunately, CMRderived parameters, as defined by the 2010 ARVC/D Task Force Criteria, may provide a major discriminatory role.

\section{Funding None.}

Conflict of interest None declared.

Open Access This article is distributed under the terms of the Creative Commons Attribution License which permits any use, distribution, and reproduction in any medium, provided the original author(s) and the source are credited.

\section{References}

1. Schölzel BE, Snijder RJ, Mager JJ, et al. Chronic thromboembolic pulmonary hypertension. Neth Heart J. 2014. doi:10.1007/s12471014-0592-2.

2. Roudijk RW, Gujic M, Suman-Horduna I, Marchese P, Ernst S. Catheter ablation in children and young adults: is there an additional benefit from remote magnetic navigation? Neth Heart J. 2013;21: 296-303. doi:10.1007/s12471-013-0408-9.

3. Groeneweg JA, van der Heijden JF, Dooijes D, van Veen TA, van Tintelen JP, Hauer RN. Arrhythmogenic cardiomyopathy: diagnosis, genetic background, and risk management. Neth Heart J. 2014;22: 316-25. doi:10.1007/s12471-014-0563-7.

4. Rebergen SA, Ottenkamp J, Doornbos J, van der Wall EE, Chin JG, de Roos A. Postoperative pulmonary flow dynamics after Fontan surgery: assessment with nuclear magnetic resonance velocity mapping. J Am Coll Cardiol. 1993;21:123-31.

5. Pattynama PM, Lamb HJ, Van der Velde EA, Van der Geest RJ, Van der Wall EE, De Roos A. Reproducibility of MRI-derived measurements of right ventricular volumes and myocardial mass. Magn Reson Imaging. 1995;13:53-63.

6. van der Wall EE, Kayser HW, Bootsma MM, de Roos A, Schalij MJ. Arrhythmogenic right ventricular dysplasia: MRI findings. Herz. 2000;25:356-64.

7. Güçlü A, Germans T, Witjas-Paalberends ER, et al. ENerGetIcs in hypertrophic cardiomyopathy: traNslation between MRI, PET and cardiac myofilament function (ENGINE study). Neth Heart J. 2013;21:567-71. doi:10.1007/s12471-013-0478-8.

8. Claessen G, Claus P, Delcroix M, Bogaert J, La Gerche A, Heidbuchel $\mathrm{H}$. Interaction between respiration and right versus left ventricular volumes at rest and during exercise: a real-time cardiac magnetic resonance study. Am J Physiol Heart Circ Physiol. 2014;306:H816-24. doi:10.1152/ajpheart.00752.2013.

9. Winter MM, Romeih S, Bouma BJ, et al. Is cardiac $\mathrm{CT}$ a reproducible alternative for cardiac MR in adult patients with a systemic right ventricle? Neth Heart J. 2012;20:456-62. doi:10.1007/s12471-0120310-x.

10. Quick S, Speiser U, Kury K, Schoen S, Ibrahim K, Strasser R. Evaluation and classification of right ventricular wall motion abnormalities in healthy subjects by 3 -tesla cardiovascular magnetic resonance imaging. Neth Heart J. 2014. doi:10.1007/ s12471-014-0620-2.

11. Grothues F, Moon JC, Bellenger NG, Smith GS, Klein HU, Pennell DJ. Interstudy reproducibility of right ventricular volumes, function, and mass with cardiovascular magnetic resonance. Am Heart J. 2004; 147:218-23.

12. Youssef A, Ibrahim e-SH, Korosoglou G, Abraham MR, Weiss RG, Osman NF. Strain-encoding cardiovascular magnetic resonance for assessment of right-ventricular regional function. J Cardiovasc Magn Reson. 2008;10:33. doi:10.1186/1532-429X-10-33.

13. von Knobelsdorff-Brenkenhoff F, Tkachenko V, Winter L, et al. Assessment of the right ventricle with cardiovascular magnetic resonance at 7 Tesla. J Cardiovasc Magn Reson. 2013;15:23. doi:10. 1186/1532-429X-15-23.

14. Doesch C, Zompolou C, Streitner F, et al. CMR-derived TAPSE measurement: a semi-quantitative method of right ventricular function assessment in patients with hypertrophic cardiomyopathy. Neth Heart J. 2014. doi:10.1007/s12471-014-0601-5.

15. Luijkx T, Velthuis BK, Prakken NH, et al. Impact of revised task force criteria: distinguishing the athlete's heart from ARVC/D using cardiac magnetic resonance imaging. Eur J Prev Cardiol. 2012;19: 885-91. doi:10.1177/1741826711414215. 